\title{
The National Early Warning Score and the acutely confused patient
}

An acute change in a person's level of consciousness can be alarming and is often an important indicator of acute-illness severity or clinical deterioration that clinicians have long recognised requires urgent clinical assessment. Indeed, NHS advice for the public is to 'get medical help immediately if someone suddenly becomes confused (delirious).'. This recognises that the onset of acute confusion may be the first indication of a significant acute perturbation to health, particularly in older patients. Thus, acute confusion is going to become an increasingly important sign of clinical deterioration as populations age.

When developing the original National Early Warning Score (NEWS), for pragmatism when assessing a patient's level of consciousness, we recommended the use of the simple and widely used AVPU scale to record whether a patient was Alert, responded to Voice, Pain or was Unresponsive. ${ }^{2}$ In updating NEWS to NEWS2, ${ }^{3}$ we took account of feedback from users of the NEWS who had noted that the existing AVPU scale might underestimate illness severity in some patients because it is possible for those recording the score at the bedside to describe a patient as alert (which would be scored at 0 points), whilst at the same time, not recognising that the patient is acutely confused (which should always have scored 3 points, even in the original NEWS). This 'confusion' about how to use the AVPU scale in an acutely confused patient who was otherwise alert, was a concern because new-onset or worsening confusion, delirium or any other altered mentation should always prompt concern about potentially serious underlying causes and warrants urgent clinical evaluation. Acutely altered mentation may occur as a consequence of many common causes in patients with acute illness, such as infections or sepsis, hypoxia, hypotension or metabolic disturbances, either alone or in combination. A recent analysis of data from over a million patient encounters, including approximately 850,000 patients with presumed infection, to determine which physiological parameters could most accurately identify patients with sepsis at the bedside found that patients with any two of three parameters, ie altered mentation (Glasgow coma score $(\mathrm{GCS})<15$ ), along with tachypnoea (respiration rate 22 breaths/minute or more), or hypotension (systolic blood pressure $100 \mathrm{mmHg}$ or less), had a high risk of clinical deterioration. ${ }^{4}$ This formed the basis of the quick sequential organ failure assessment (qSOFA) score which was subsequently shown to predict mortality in patients presenting with suspected infection, significantly more accurately than conventional descriptors of 'severe sepsis'. Indeed, mortality was $3 \%$ for patients with qSOFA scores of 0 or 1 , but $24 \%$ for patients with a qSOFA score of 2 or more. ${ }^{5}$ Thus, acute confusion is a potent marker of clinical risk and acute illness severity and could be missed by simple recording of the AVPU scale, unless the possibility of new confusion is routinely considered. This prompted the addition of ' $C$ ' to the new ACVPU scale in which any deviation from a normal alert state (which would correspond to a GCS $<15$ ), is recorded as a score of 3 on the NEWS 2 chart. ${ }^{3}$ Because it is not always possible to determine whether confusion is 'new', we recommended that any confusion in an acutely ill patient should always be considered to be 'new' until confirmed to be otherwise. Mindful of the clear evidence that new confusion is an indicator of potentially severe physiological disturbance and risk of clinical deterioration, we recommended that these patients are promptly evaluated to establish the cause and initiate treatment. ${ }^{3}$

In this issue of Clinical Medicine, Mohammed et al, report a study in which they have randomly assigned $20 \%$ of acute admissions to have delirium, at two acute hospitals. ${ }^{6}$ They then assessed the impact of this high level of acute delirium in acutely ill patients, on the number of medium and high level NEWS2 alerts. Not surprisingly, they found that these alerts would increase. The authors concluded that in light of this entirely predictable but simulated finding, that rigorous evaluation of the NEWS2 is required before implementation, to study its potentially adverse effect on workload. This raises two important questions. First, is it being suggested that acutely ill patients who have developed an acute alteration in mental state and have become acutely confused or delirious should not be responded to urgently? Surely not, mindful of the high risk of many of these patients and the existing advice from the NHS. ${ }^{1}$ Or, is the question more about whether alert systems that identify patients who need an urgent response should be adjusted to reflect the capacity of the system to respond? This would mean that the denominator for change is 'what works for the system' rather than the patients the system is designed to serve. Moreover, it means that advances in care would never keep pace with advances in medical science. This is not to undermine the legitimate concern about the impact of system change on the changing demands imposed on the staff working in front-line acute services in the NHS, I work there myself. On the contrary, the widespread use of the NEWS and now NEWS2 has identified and prompted the need for changes in the configuration of the acute care workforce to enable a more timely detection and appropriate response to acutely ill patients 24/7. Ensuring that innovation drives the system rather than the system resisting innovation. Patients with acute confusion have always needed an urgent clinical response. NEWS2 hasn't created the workload, it has simply identified what was always there and that some systems of care are not always configured to respond in the way that they should.

BRYAN WILLIAMS

Chair of medicine, University College London, London, UK Chairman of the NEWS and NEWS2 Development

Groups for the RCP 


\section{References}

1 National Health Service. Sudden confusion (delirium). NHS, 2018. www.nhs.uk/conditions/confusion [Accessed 18 February 2019].

2 Royal College of Physicians. National Early Warning Score (NEWS): Standardising the assessment of acute illness severity in the NHS. Report of a working party. London: RCP, 2012.

3 Royal College of Physicians. National Early Warning Score (NEWS) 2: Standardising the assessment of acute-illness severity in the NHS. Updated report of a working party. London: RCP, 2017.
4 Seymour CW, Liu VX, Iwashyna T] et al. Assessment of clinical criteria for sepsis: for the Third International Consensus Definitions for Sepsis and Septic Shock (Sepsis-3). JAMA 2016;315:762-74.

5 Freund $\mathrm{Y}$, Lemachatti N, Krastinova E et al. Prognostic accuracy of sepsis-3 criteria for in-hospital mortality among patients with suspected infection presenting to the emergency department. JAMA 2017:317:301-8.

6 Mohammed M, Faisal M, Richardson D et al. The inclusion of delirium in version 2 of the National Early Warning Score will substantially increase the alerts for escalating levels of care: findings from a retrospective database study of emergency medical admissions in two hospitals. Clin Med 2019;19:104-8.

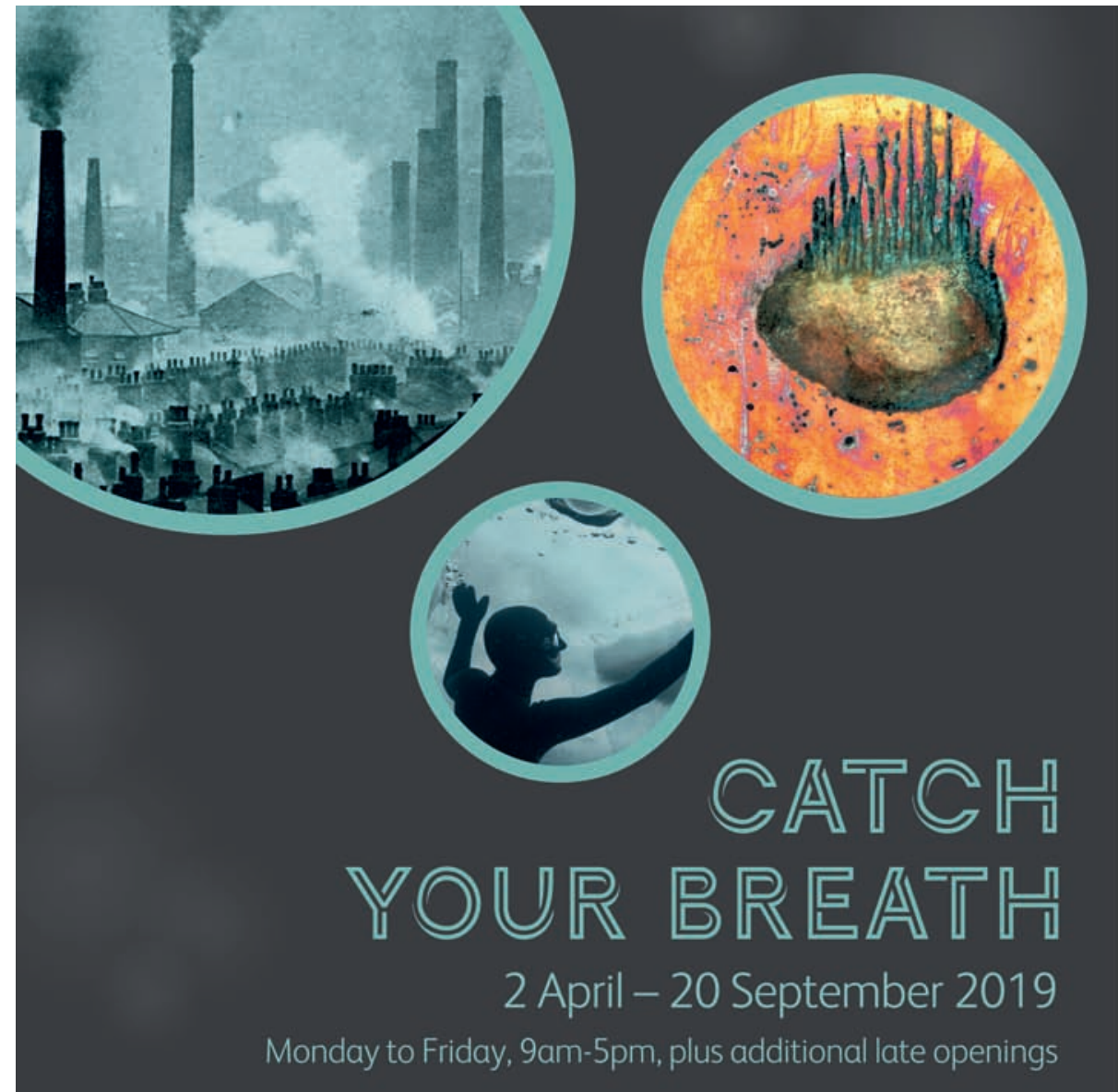

\section{TEMPORARY EXHIBITION}

Royal College of Physicians 11 St Andrews Place Regent's Park London NW1 4LE

\#CatchYourBreath @RCPmuseum @LifeofBreath history.rcplondon.ac.uk/events catchyourbreath.org

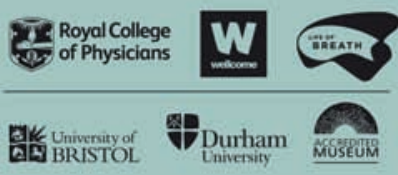

